\title{
Comprehensive Effects of Suppression of MicroRNA-383 in Human Bone-Marrow- Derived Mesenchymal Stem Cells on Treating Spinal Cord Injury
}

\author{
Guo-Jun Wei Ke-wen Zheng $^{\mathrm{b}}$ Gang An ${ }^{\mathrm{a}}$ Zuo-Wei Shia Kai-Fu Wang ${ }^{\mathrm{a}}$ \\ Ying Guan ${ }^{a}$ Yan-Song Wang ${ }^{a}$ Peng-Fei Lia Da-Ming Dong ${ }^{a}$ \\ aDepartment of Orthopaedics, the 1st Affiliated Hospital of Harbin Medical University, Harbin, \\ ${ }^{b}$ Department of Orthopedics, Hongqi Hospital of Mudanjiang Medical University, Mudanjiang, China
}

\section{Key Words}

Glial cell line derived neurotrophic factor (GDNF) • Mesenchymal stem cells (MSCs) • MiR-383 - Spinal cord injury (SCI) - Vascular endothelial growth factor A (VEGF-A) - Cyclin-dependent kinase 19 (CDK19)

\footnotetext{
Abstract

Background/Aims: Transplantation of bone-marrow-derived mesenchymal stem cells (MSCs) promotes neural cell regeneration after spinal cord injury (SCI). Recently, we showed that suppression of microRNA-383 (miR-383) in MSCs increased the protein levels of glial cell line derived neurotrophic factor (GDNF), resulting in improved therapeutic effects on SCI. However, the overall effects of miR-383 suppression in MSCs on SCI therapy were not determined yet. Here, we addressed this question. Methods: We used bioinformatics tools to predict all miR383-targeting genes, confirmed the functional bindings in a dual luciferase reporter assay. The effects of alteration of candidate genes in MSCs on cell proliferation were analyzed by MTT assay and by Western blotting for PCNA. The effects on angiogenesis were assessed by HUVEC assay. The effects on SCI in vivo were analyzed by transplantation of the modified MSCs into nude rats that underwent SCI. Results: Suppression of miR-383 in MSCs not only upregulated GDNF protein, but also increased vascular endothelial growth factor A (VEGF-A) and cyclin-dependent kinase 19 (CDK19), two other miR-383 targets. MiR-383-suppressioninduced increases in CDK19 resulted in a slight but significant increase in MSC proliferation, while miR-383-suppression-induced increases in VEGF-A resulted in a slight but significant increase in MSC-mediated angiogenesis. Conclusions: Upregulation of CDK19 and VEGF-A by miR-383 suppression in MSCs further improve the therapeutic potential of MSCs in treating $\mathrm{SCI}$ in rats.

G.-J. Wei and K.-w. Zheng contributed equally to this work.

Da-Ming Dong

Department of Orthopaedics, The 1st Affiliated Hospital of Harbin Medical University

23 Youzheng Street, Harbin 150001 (China)

Tel.+8645185555233; E-Mail dongdaming1965@hotmail.com
}

KARGER 


\section{Introduction}

Owing to the potential effects on tissue repair, transplantation of bone-marrow-derived mesenchymal stem cells (MSCs) has been used in treating a variety of diseases [1-3], including spinal cord injury (SCI), in which the neural cell regeneration was compensated by MSC grafting [4-6].

Glial cell line derived neurotrophic factor (GDNF) is critical for neural growth and survival, and appears to be an essential trophic factor for development of enteric, sympathetic and parasympathetic neurons [7]. Interestingly, GDNF has been overexpressed in MSCs to enhance their therapeutic effects in a fatal neurodegenerative disease called amyotrophic lateral sclerosis (ALS) [8]. In this study, the authors showed that engineered MSCs that secreted GDNF significantly increased the number of neuromuscular connections and motor neuron cell bodies in the spinal cord in the disease progression, and ameliorate motor neuron loss within the spinal cord, resulting in a significant delay of disease progression and a significant increase in overall lifespan of the experimental rats [8]. Since neural cell regeneration is critical for post-SCI recovery, we have examined the effects of overexpressing GDNF in MSCs on the therapeutic outcome for SCI [9]. Different from direct expression of GDNF in MSCs, we suppressed a GDNF-targeting microRNA, miR-383, in MSCs to enhance GDNF protein production in MSCs, resulting in a therapeutic potential comparable to direct GDNF expression. Since GDNF in MSCs is regulated by miR-383-mediated post-transcriptional control, we think that removal or dismissal of this post-transcriptional control that has a lower chance to cause ER stress should be advantageous over overwhelmingly expression of high GDNF [8, 9]. Our previous report thus highly suggest that suppression of miR-383 may increase the therapeutic potential of human MSCs in treating SCI via augmentation of GDNF protein levels [9]. However, there is an important remaining question.

As non-coding small RNAs that regress mRNA translation through Watson-Crick pairing to the $3^{\prime}$-untranslated region (3'-UTR) of the target mRNA, miRNAs typically have a number of target mRNA from a variety of genes [10-13]. Therefore, it is still unknown which genes other than GDNF may be affected by miR-383 suppression in MSCs, and how modulation of non-GDNF genes may affect the therapeutic effects of MSCs on SCI. Here we addressed this question.

In the current study, we used bioinformatics tools to predict miR-383-targeting genes other than GDNF and examined their influence on the outcome of MSCs in treating SCI.

\section{Materials and Methods}

\section{Protocol approval}

All the experimental methods including animal protocols in the current study have been approved by the research committee at Harbin Medical University and carried out in accordance.

\section{Production of adeno-associated viruses for MSC transduction}

The Human Embryonic Kidney 293 cell line (HEK293, American Type Culture Collection, ATCC, Rockville, MD, USA) was used for virus production, as previously reported [9]. We used a pAAV-CMVp-GFP plasmid (Clontech, Mountain View, CA, USA), a packaging plasmid R2C8 carrying the serotype 2 rep and cap genes (Applied Viromics, LLC. Fremont, CA, USA), and a helper plasmid plAd5 carrying the adenovirus helper functions (Applied Viromics, LLC.) for generating AAVs in this study. AAVs were produced by cotransfecting HEK293 cells with the prepared pAAV-CMVp-as-miR-383 or pAAV-CMVp-Null plasmids, and a shRNA for CDK19 (shCDK19) or a shRNA for VEGF-A (shVEGF-A), R2C8 and plAd5 by Lipofectamine 3000 (Invitrogen, St. Louis, MO, USA). The viruses were purified using CsCl density centrifugation and then titration was determined by a quantitative densitometric dot-blot assay. These viruses were used to transduce MSCs using a MOI of 20 to 100. 


\section{Cellular Physiology Cell Physiol Biochem 2018;47:129-139

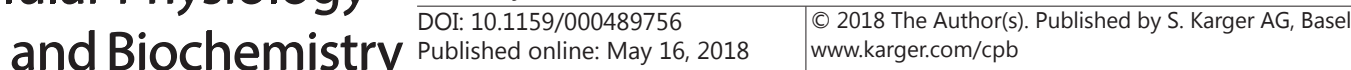

Wei et al.: MiR-383-Depleted Mscs in Treating SCI

\section{Cell isolation and culture}

Human bone-marrow derived MSCs were isolated from a 40-year-old male healthy donor, who had received and signed proper informed consent form after IRB approval was obtained, as previously reported [9]. Culture of MSCs was done as previously reported [9]. Human umbilical vein endothelial cells (HUVECs) were purchased from ATCC, and grown in M-200 supplemented medium with low serum growth supplement (Invitrogen).

\section{SCI model and MSC transplantation}

SCI was induced in male nude rats (SLAC Laboratory Animal, Shanghai, China) of 200g body weight, as previously reported [9]. Briefly, the 12-week-old nude rats were deeply anesthetized by intraperitoneal injection of chloral hydrate $(70 \mathrm{mg} / \mathrm{ml}, 0.4 \mathrm{ml}$ per $100 \mathrm{~g}$, Sigma-Aldrich). After skin incision, laminectomy at the Th8 vertebral level was performed. The impact rod (diameter $2 \mathrm{~mm}$, weight $10 \mathrm{~g}$ ) of an impactor was centered above Th8 and dropped from a height of $25 \mathrm{~mm}$ to induce SCI. Immediately after laminectomy or SCI, MSCs were intraspinally injected at $10^{6} / 500 \mu \mathrm{l}$ into the rats, using a $25 \mathrm{G}$ needle. To avoid significant flowback, the injection was performed in a very slow speed (about $10 \mu \mathrm{l} / \mathrm{second}$ ) at one spot, after which gel foam thrombin (Pfizer, Shanghai, China) was applied onto the injection spot after injection. After surgery, $0.05 \mathrm{mg} / \mathrm{kg}$ buprenorphine was administered subcutaneously for analgesia and the animals were monitored for $1 \mathrm{~h}$ before they were transferred back and maintained in a specific pathogen-free environment in positive pressure rooms with a standard 12-h day/12-h night cycle.

\section{Quantitative real-time PCR (RT-qPCR)}

Total RNA were extracted from cultured cells with miRNeasy mini kit (Qiagen, Hilden, Germany) for cDNA synthesis. Quantitative real-time PCR (RT-qPCR) was performed in duplicates with QuantiTect SYBR Green PCR Kit (Qiagen). All primers were purchased from Qiagen. Data were collected and analyzed using $2-\Delta \Delta \mathrm{Ct}$ method. Values of genes were first normalized against $\alpha$-tubulin, and then compared to experimental controls.

\section{Western blot}

Proteins were isolated from cultured cells using RIPA lysis buffer (Sigma-Aldrich). Protein concentration was determined using a BCA protein assay kit (Bio-rad, Shanghai, China), and the cell lysates were mixed with $4 \times$ SDS loading buffer (Bio-rad), heated, and then separated on SDS-polyacrylamide gels. After transferred to a PVDF membrane, membrane blots were first probed with a primary antibody, and then horseradish peroxidase-conjugated second antibody, to be photographed with enhanced chemiluminescent system. Primary antibodies were rabbit anti-GDNF, anti-CDK19 and anti- $\alpha$-tubulin (Cell Signaling, Carpinteria, CA, USA). Secondary antibody is HRP-conjugated anti-rabbit (Dako, Carpinteria, CA, USA). Fig. images were representative from 5 repeats. $\alpha$-tubulin was used as a protein loading control.

\section{Luciferase-reporter activity assay}

The target genes of miR-383 was predicted by TargetScan, using the context++ score system, as described [14]. The dual-luciferase reporter plasmids, p3'-UTR-CDK19 (containing the wild-type CDK19 3'-UTR binding site in luciferase reporter plasmid (RiboBio Co. Ltd., Shanghai, China)), p3'-UTR-CDK19mut (containing a mutant CDK19 3'-UTR at the miR-383 binding site; mut), p3'-UTR-VEGF-A (containing the wild-type VEGF-A 3'-UTR binding site in luciferase reporter plasmid (RiboBio Co. Ltd.) and p3'-UTRVEGF-A-mut (containing a mutant VEGF-A 3'-UTR at the miR-383 binding site; mut) as well as miR-383, antisense of miR-383 (as-miR-383) and a null control were constructed in RiboBio Co. Ltd. For the luciferase assay, the constructed 3'-UTR plasmid (500ng) and miR-383/as-miR-383 (100 nmol/l) were co-transfected into Human MSCs using LipofectamineTM 3000 Reagent (Invitrogen, Shanghai China). Then the luciferase activity was detected with the dual-luciferase reporter assay system (Promega, Shanghai, China) after cotransfection cells for 48 hours, following the manufacturer's protocol.

\section{Histological assessment}

For histological assessment, rats were anesthetized with chloral hydrate, prior to intracardiac perfusion with 4\% paraformaldehyde (PFA, Sigma-Aldrich) 28 days after transplantation. The tissue was resected and fixed in PFA for 6 hours. After overnight incubation in 30\% sucrose, samples were frozen in liquid nitrogen 


\section{Cellular Physiology Cell Physiol Biochem 2018;47:129-139 \begin{tabular}{l|l} 
DOI: 10.1159/000489756 & and Biochemistry \\
Published online: May 16, 2018 & $\begin{array}{l}\text { O 2018 The Author(s). Published by S. Karger AG, Basel } \\
\text { www.karger.com/cpb }\end{array}$
\end{tabular} \\ Wei et al.: MiR-383-Depleted Mscs in Treating SCI}

and embedded in tissue freezing medium. Non-fixed tissue was used for RT-qPCR and Western blot. Twenty micron longitudinal tissue sections were then stained with Azur-eosin for measurement of tissue and cavity volume as described previously [9]. Briefly, every fifth section was selected from the consecutive sectioning and its high-resolution image was used to identify areas using ImageJ software (NIH, Bethesda, MA, USA). Any necrotic tissue within the cavities was counted as part of the lesion. The cavity volume was assessed as the sum of section areas multiplied by the distance between them, and the total spinal cord volume of the segment was also measured. The combined area of any cysts was calculated and subtracted from the total tissue area of intact four section to estimate total tissue remaining. Ten rats were used in each experimental group.

\section{Behavioral assessment}

Locomotor recovery was evaluated using the open-field BBB locomotor ratio scale, as described before [9]. The motor functions of the lower limbs of the rats were evaluated in a blind way. For the scoring system, the motor functions of the lower limbs are divided into 22 grades. While 0 points indicate hind leg paralysis, 21 indicates completely normal function. The BBB score observation period lasted for $4 \mathrm{~min}$.

\section{MTT assay}

For assay of cell number, $10^{4}$ cells were seeded into 24 well-plate at 10000 cells per well and subjected to a Cell Proliferation Kit (MTT, Roche, Indianapolis, IN, USA), according to the instruction of the manufacturer. The insoluble tetrazolium dye 3-(4, 5-dimethylthiazol-2-yl)-2, 5-diphenyltetrazolium bromide (MTT) form was quantified by absorbance value (OD) at $570 \mathrm{~nm}$. Experiments were performed 5 times.

\section{ELISA}

Protein was extracted from the cultured cells or conditioned media by RIPA buffer (Sigma-Aldrich St. Louis, MO, USA). ELISA for VEGF-A was performed using commercial ELISA kits (R\&D Systems, Minneapolis, $\mathrm{MN}, \mathrm{USA}$ ), according to the manufacturer's instruction. The absorption was measured at $450 \mathrm{~nm}$. The protein concentration was determined by comparing the relative absorbance of the samples with the standards.

\section{HUVEC collagen gel assay}

HUVEC endothelial cells (ATCC) were embedded in a collagen gel, and plated on a 24-well culture plate. The plate was kept at $37^{\circ} \mathrm{C}$ in a $\mathrm{CO}_{2}$ incubator for 15 minutes to make the collagen polymerize, and then the same number of MSCs (MSCs-null, or MSCs-as-miR-383, or MSCs-as-miR-383-shVEGF-A) was added to the transwell and put onto the plate. The plate with transwell was returned to the $\mathrm{CO}_{2}$ incubator. Media were replenished every day, and culture images were taken and the vessel formation of HUVECs was quantified after 3 days.

\section{Statistical analysis}

All values represent the mean \pm standard deviation (SD). Statistical analysis of group differences was carried out using a one-way analysis of variance (ANOVA) test (SPSS 12.0, Chicago, IL, USA) followed by the Fisher's Exact Test to compare two groups. Ten rats were used in each experimental group. A value of $p<0.05$ was considered statistically significant after Bonferroni correction.

\section{Results}

Besides GDNF, miR-383 also targets 3'-UTR of CDK19 and VEGF-A mRNA to inhibit protein translation in MSCS

We have previously suppressed miR-383 in human MSCs, through expression of asmiR-383 in an AAV-mediated transduction system. Now, we analyzed genes other than GDNF that may be affected by miR-383 suppression in MSCs, and how modulation of non-GDNF genes may affect the therapeutic effects of MSCs on SCI.

Thus, we performed bioinformatics analyses to identify miR-383 targeting mRNAs. A number of genes were predicted. From these candidates, we only found that the protein levels, but not mRNA levels of cyclin-dependent kinase 19 (CDK19) and vascular endothelial 
Fig. 1. MiR-383 also targets 3'-UTR of CDK19 and VEGF-A mRNA to inhibit protein translation in MSCs. (A) RT-qPCR for CDK19 and VEGF-A in MSCs-asmiR-383 and MSCs-null. (B) Western blotting for CDK19 in MSCs-as-miR-383 and MSCs-null. (C) ELISA for VEGF-A in MSCs-as-miR-383 and MSCs-null. (D) Bioinformatics analyses show the binding site of miR-383 on the 3'-UTR of CDK19 mRNA. (E) Bioinformatics analyses show the binding site of miR-383 on the 3'-UTR of VEGF-A mRNA. (F) The functional binding of miR-383 to CDk19 mRNA was then examined in a dual luciferase reporter assay. The intact 3'UTR of wildtype CDK19 mRNA (CDK19 3'-UTR) and the 3'-UTR of CDK19 mRNA with a mutant at miR-383-binding site (CDK19 3'-UTR mut) were respectively cloned into luciferase reporter plasmids. MSCs were then co-transfected with one plasmid from miR-383/asmiR-383/null plasmids and one plasmid from either CDK19 3'-UTR or CDK19 3'UTR mut, and subsequently subjected

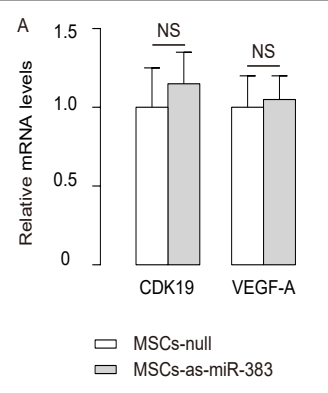

D Binding of miR-383 onto 3'-UTR of CDK19 mRNA E Binding of miR-383 onto 3'-UTR of VEGF-A mRNA CDK19 3'-UTR (250-256)

5' UUCUCUGAUAAAGCGUCUGAUCU 3 ' | |||| $\mid$ 3' UCGGUGUUAGUGGAAGACUAGA 5'
miR-383

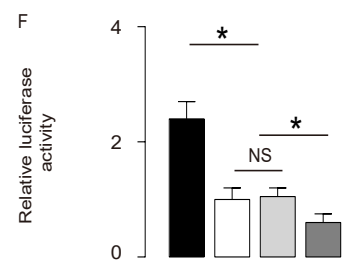
as-miR-383 miR-383 - - + + CDK19 3'-UTR mut - - + -
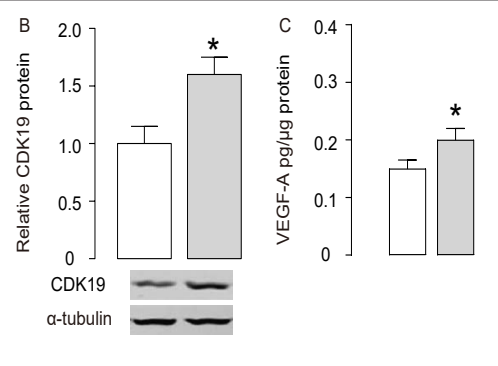
5' AUUAAAGAAUUUAAUUCUGAUCU 3' II| |||||| $\mid$ 3' UCGGUGUUAGUGGAAGACUAGA 5'

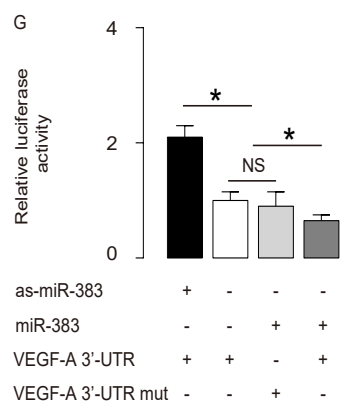
to a dual luciferase reporter assay. The luciferase activity was determined. (G) The intact 3'-UTR of wildtype VEGF-A mRNA (VEGF-A 3'-UTR) and the 3'-UTR of VEGF-A mRNA with a mutant at miR-383-binding site (VEGF-A 3'-UTR mut) were respectively cloned into luciferase reporter plasmids. MSCs were then co-transfected with one plasmid from miR-383/ as-miR-383/null plasmids and one plasmid from either VEGF-A 3'-UTR or VEGF-A 3'-UTR mut, and subsequently subjected to a dual luciferase reporter assay. ${ }^{*} \mathrm{p}<0.05$. NS: non-significant. $\mathrm{N}=5$.

growth factor A (VEGF-A) were significantly increased by miR-383 suppression (Fig. 1A-C), which was attributable to the direct binding of miR-383 onto the 3'-UTR of CDK19 mRNA (Fig. 1D) and VEGF-A mRNA (Fig. 1E), respectively.

The functional binding of miR-383 to CDk19 and VEGF-A mRNA was then examined in a dual luciferase reporter assay. The intact 3'-UTR of wildtype CDK19 mRNA (CDK19 3'-UTR) and the 3'-UTR of CDK19 mRNA with a mutant at miR-383-binding site (CDK19 3'-UTR mut) were respectively cloned into luciferase reporter plasmids. MSCs were then co-transfected with one plasmid from miR-383/as-miR-383/null plasmids and one plasmid from either CDK193'-UTR or CDK193'-UTR mut, and subsequently subjected to a dual luciferase reporter assay. We found that depletion of miR-383 increased luciferase activity of CDK19 3'-UTR, while overexpression of miR-383 reduced luciferase activity of CDK19 3'-UTR but had no effects on CDK19 3'-UTR mut. These results suggest that miR-383 specifically targets 3'-UTR of CDK19 mRNA to inhibit its translation (Fig. 1F). Next, the intact 3'-UTR of wildtype VEGF-A mRNA (VEGF-A 3'-UTR) and the 3'-UTR of VEGF-A mRNA with a mutant at miR-383-binding site (VEGF-A 3'-UTR mut) were respectively cloned into luciferase reporter plasmids. MSCs were then co-transfected with one plasmid from miR-383/as-miR-383/null plasmids and one plasmid from either VEGF-A 3'-UTR or VEGF-A 3'-UTR mut, and subsequently subjected to a dual luciferase reporter assay. We found that depletion of miR-383 increased luciferase activity of VEGF-A 3'-UTR, while overexpression of miR-383 reduced luciferase activity of VEGF-A 3'-UTR but had no effects on VEGF-A 3'-UTR mut. These results suggest that miR- 
383 specifically targets 3'-UTR of VEGF-A mRNA to inhibit its translation (Fig. 1G). Thus, besides GDNF, miR-383 also targets 3'-UTR of CDK19 and VEGF-A mRNA to inhibit protein translation in MSCs. Since CDK19 functions in cell proliferation and VEGF-A functions as a pro-angiogenic factor, we analyzed the effects of their upregulation in MSCs by miR-383 suppression on the growth and pro-angiogenic effects of MSCs in vitro, as well as the effects of their upregulation in MSCs upon therapeutic potential in SCI.

Upregulation of CDK19 in MSCs by miR-383 suppression increases MSC growth in vitro

First, the effects of upregulation of CDK19 by miR-383 suppression in MSCs on cell growth were analyzed in vitro. In order to confirm that the effects on cell growth are exactly from miR-383-suppression-induced CDK19 upregulation, shCDK19 was used to transduce MSCs-as-miR-383 as a loss-of-function control (MSCs-as-miR-383-shCDK19). We found that CDK19 mRNA was not affected by miR-383 suppression (Fig. 2A), but CDK19 protein was significantly increased by miR-383 suppression (Fig. 2B), consistent with previous results (Fig. 1A-B). Suppression of CDK19 by shCDK19 in MSCs-as-miR-383 directly reduced CDK19 mRNA, and abolished the increases in CDK19 protein in MSCs-as-miR-383, compared to MSCs-null controls (Fig. 2A-B). Moreover, neither GDNF mRNA nor GDNF protein levels were altered by shCDK19 in MSCs-as-miR-383 (Fig. 2A-B). Hence, MSCs-as-miR-383-shCDK19 appeared to be a good control to evaluate the effects of CDK19 upregulation in MSCs by miR383 suppression on the growth of MSCs in vitro, as well as the effects of CDK19 upregulation by miR-383 suppression in MSCs upon therapeutic potential in SCI.

Cell proliferation potential was analyzed by Western blotting for PCNA and in a MTT assay in vitro. We found that miR-383 suppression slightly but significantly increased the PCNA levels in MSCs, which was abolished by direct suppression of CDK19 by shCDK19, suggesting that the effects on cell proliferation by miR-383 suppression are exactly from induced CDK19 upregulation (Fig. 2C). Moreover, miR-383 suppression slightly but significantly increased the in vitro cell growth in MSCs in an MTT assay, which was abolished by direct suppression of CDK19 by shCDK19, suggesting that the effects on cell growth by miR-383 suppression are exactly from induced CDK19 upregulation (Fig. 2D). Together, these data suggest that upregulation of CDK19 in MSCs by miR-383 suppression increases MSC growth in vitro.

Fig. 2. Upregulation of CDK19 in MSCs by miR-383 suppression increases MSC growth in vitro. The effects of upregulation of CDK19 by miR-383 suppression in MSCs on cell growth were analyzed in vitro. In order to confirm that the effects on cell growth are exactly from miR383-suppression-induced CDK19 upregulation, shCDK19 was used to transduce MSCs-as-miR-383 as a loss-of-function control (MSCsas-miR-383-shCDK19). (A) RTqPCR for CDK19 and GDNF in MSCs-as-miR-383, MSCs-as-miR383-shCDK19 and MSCs-null. (B) Western blotting for CDK19 and GDNF in MSCs-as-miR-383, MSCs-as-miR-383-shCDK19 and MSCs-null. (C) Western blotting for PCNA in MSCs-as-miR-383, MSCs-as-miR-383-shCDK19 and MSCs-null. (D) MTT assay for MSCs-as-miR-383, MSCs-as-miR-383-shCDK19 and MSCs-null. ${ }^{*} \mathrm{p}<0.05$. NS: non-significant. $\mathrm{N}=5$.
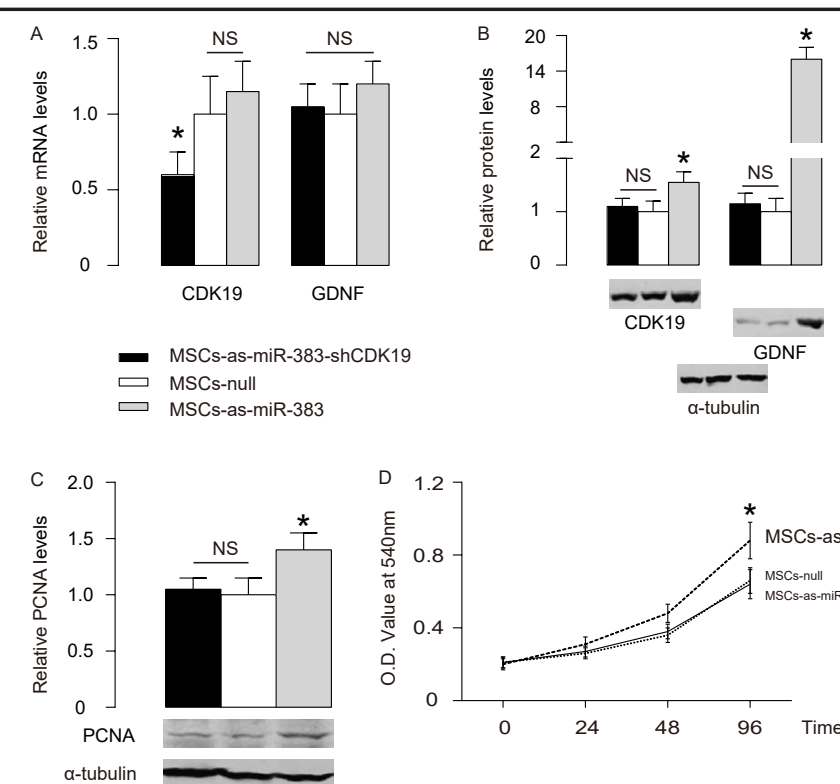

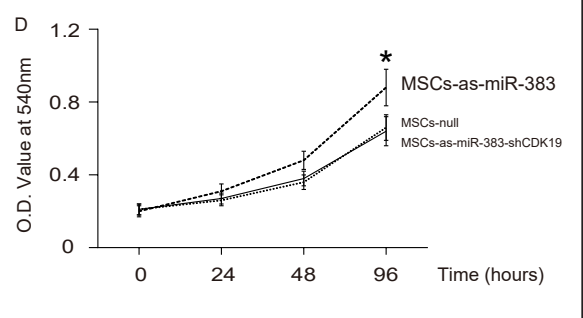


Fig. 3. Upregulation of VEGF-A in MSCs by miR-383 suppression increases pro-angiogenic potential of MSCs in vitro. The effects of upregulation of VEGF-A by miR-383 suppression on pro-angiogenic potential of MSCs were analyzed in vitro. In order to confirm that the effects on pro-angiogenic potential of MSCs are exactly from miR383-suppression-induced VEGF-A upregulation, shVEGF-A was used to transduce MSCs-as-miR-383 as a loss-of-function control (MSCs-asmiR-383-shVEGF-A). (A) RT-qPCR for VEGF-A and GDNF in MSCs-asmiR-383, MSCs-as-miR-383-shVEGF-A and MSCs-null. (B) ELISA for VEGF-A in MSCs-as-miR-383, MSCs-as-miR-383-shVEGF-A and MSCs-null. (C) Western blotting for GDNF in MSCs-as-miR-383, MSCs-as-miR-383-shVEGF-A and MSCs-null. (D-E) A HUVEC transwell collagen gel assay for MSCsas-miR-383, MSCs-as-miR-383-shVEGF-A and MSCs-null, shown by quantification (D), and by representative images (E). ${ }^{*} \mathrm{p}<0.05$. NS: non-significant. $\mathrm{N}=5$. Scale bars are $50 \mu \mathrm{m}$.

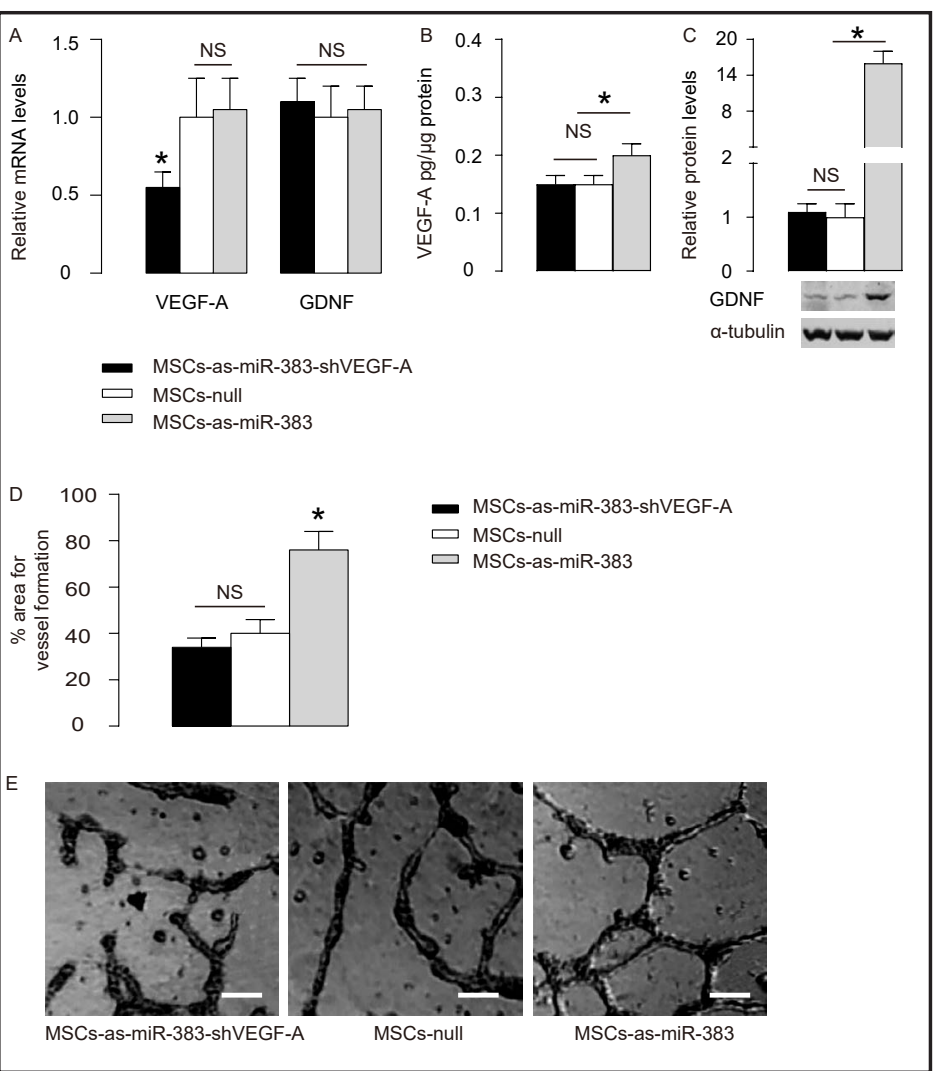

Upregulation of VEGF-A in MSCs by miR-383 suppression increases pro-angiogenic potential of MSCS in vitro

Next, the effects of upregulation of VEGF-A by miR-383 suppression on pro-angiogenic potential of MSCs were analyzed in vitro. In order to confirm that the effects on pro-angiogenic potential of MSCs are exactly from miR-383-suppression-induced VEGF-A upregulation, shVEGF-A was used to transduce MSCs-as-miR-383 as a loss-of-function control (MSCsas-miR-383-shVEGF-A). We found that neither GDNF mRNA nor GDNF protein levels were altered by shVEGF-A in MSCs-as-miR-383 (Fig. 3A-B). Moreover, VEGF-A mRNA was not affected by miR-383 suppression (Fig. 3A), but VEGF-A protein was slightly and significantly increased by miR-383 suppression (Fig. 3C), consistent with the previous results (Fig. 1A and 1C). Suppression of VEGF-A by shVEGF-A in MSCs-as-miR-383 directly reduced VEGF-A mRNA, and abolished the increases in VEGF-A protein in MSCs-as-miR-383, compared to MSCs-null controls (Fig. 3A, 3C). Moreover, Hence, MSCs-as-miR-383-shVEGF-A appeared to be a good control to evaluate the effects of VEGF-A upregulation in MSCs by miR-383 suppression on the pro-angiogenic potential of MSCs in vitro, as well as the effects of VEGF-A upregulation by miR-383 suppression in MSCs upon therapeutic potential in SCI.

Thus, a HUVEC transwell collagen gel assay was used to determine the pro-angiogenic potential of the modified MSCs. We found that miR-383 suppression in MSCs significantly increased vessel formation of co-cultured HUVEC, and this effect was abolished by direct suppression of VEGF-A by shVEGF-A on MSCs. These data suggest that the effects on the pro-angiogenic potential of MSCs-as-miR-383 may stem from the enhanced VEGF-A levels through miR-383 suppression, shown by quantification (Fig. 3D), and by representative images (Fig. 3E). Together, these data suggest that upregulation of VEGF-A in MSCs by miR383 suppression increases pro-angiogenic potential of MSCs in vitro. 
Upregulation of CDK19 and VEGF-A by miR-383 suppression in MSCs does not harm the therapeutic potential of MSCs in treating SCI in rats

Finally, we examined the effects of upregulation of CDK19 and VEGF-A by miR-383 suppression in MSCs on the therapeutic potential of MSCs in treating SCI in a rat model for SCI. SCI was induced in a nude rat. Immediately after laminectomy or SCI, MSCs-null, or MSCsas-miR-383, or MSCs-as-miR-383-shCDK19, or MSCs-as-miR-383-shVEGF-A were injected intraspinally. Another control was sham-treated without induction of SCI. The rats were then followed up for 28 days (Fig. 4A) and histology was assessed (Fig. 4B). Consistent with our previous report, we found that miR-383-depletion in MSCs further increased the intact tissue percentage (Fig. 4C), decreased cavity volume (Fig. 4D), and enhanced the recovery of locomotor activity in nude rats that underwent SCI (Fig. 4E), compared to null-transduced MSCs. Moreover, CDK19 suppression in MSCs-as-miR-383 did not alter the effects of miR-383 suppression on intact tissue percentage (Fig. 4C), cavity volume (Fig. 4D), and the recovery of locomotor activity in nude rats that underwent SCI (Fig. 4E), suggesting that the effects of increases in MSCs-as-miR-383 growth by CDK19 upregulation do not harm the therapeutic effects of MSCs-as-miR-383 on SCI. Furthermore, VEGF-A suppression in MSCs-as-miR-383 did not alter the effects of miR-383 suppression on intact tissue percentage (Fig. 4C), cavity volume (Fig. 4D), and the recovery of locomotor activity in nude rats that underwent SCI (Fig. 4E), suggesting that the effects of increases in the pro-angiogenic potential of MSCs-as-

Fig. 4. Upregulation of CDK19 and VEGF-A by miR-383 suppression in MSCs does not harm the therapeutic potential of MSCs in treating SCI in rats. (A) SCI was induced in a nude rat. Immediately after laminectomy or SCI, MSCs-null, or MSCsas-miR-383, or MSCsas-miR-383-shCDK19, or MSCs-as-miR-383shVEGF-A were injected intraspinally. Another control was sham-treated without induction of SCI. The rats were then followed up for 28 days. (B) Representative histology. (C) Intact tissue percentage. (D) Cavity volume. (E) The assessment of locomotor activity by BBB method. ${ }^{*} \mathrm{p}<0.05 . \mathrm{N}=10$. Scale bars are $50 \mu \mathrm{m}$.

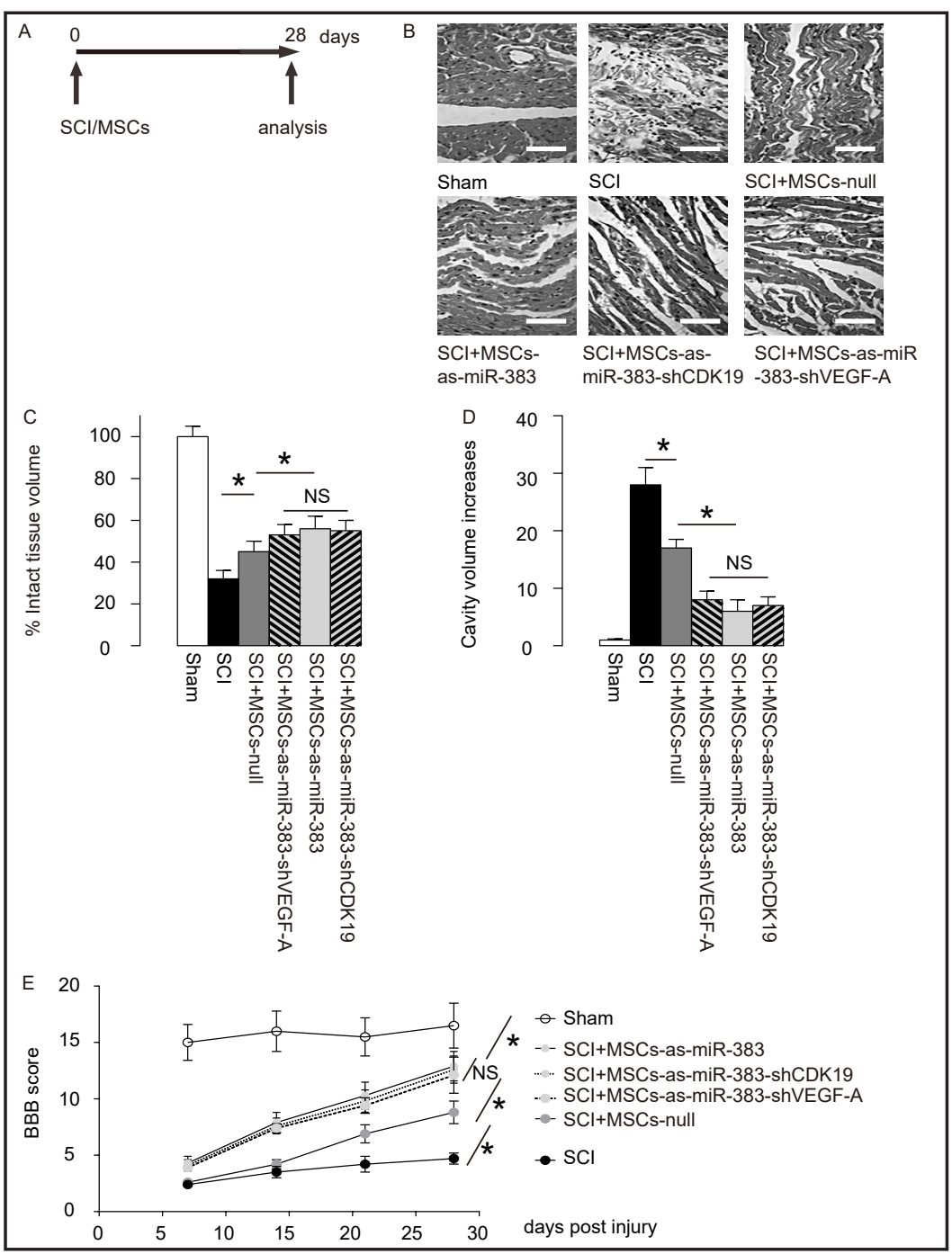




\section{Cellular Physiology Cell Physiol Biochem 2018;47:129-139 \begin{tabular}{l|l} 
DOI: 10.1159/000489756 & O 2018 The Author(s). Published by S. Karger AG, Basel \\
www.karger.com/cpb
\end{tabular} \\ Wei et al.: MiR-383-Depleted Mscs in Treating SCI}

Fig. 5. In vivo survival and limited expansion of transplanted MSCs. In order to understand the survival and expansion of transplanted MSCs, we extracted RNA from the moues spinal tissue that had received MSCs at different time courses and examined the expression of GFP, a transgene present in the transplanted MSCs, but not in host cells. RT-qPCR was

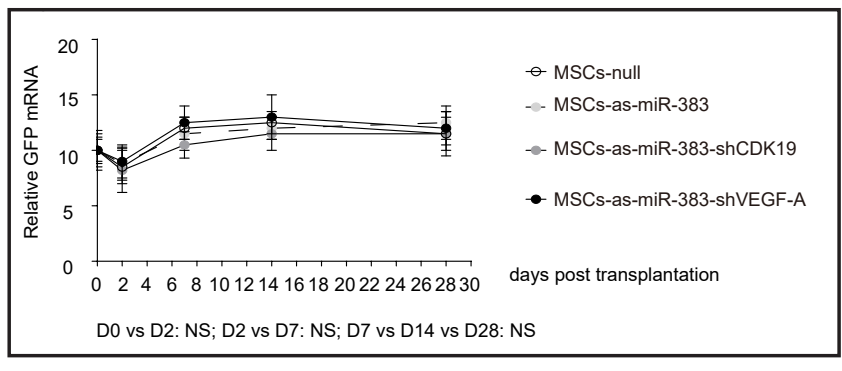
performed. NS: non-significant. $\mathrm{N}=5$.

miR-383 that upregulates VEGF-A do not harm the therapeutic effects of MSCs-as-miR-383 on SCI. Together, these data suggest that upregulation of CDK19 and VEGF-A by miR-383 suppression in MSCs does not harm the therapeutic potential of MSCs in treating SCI in rats. No further adaptation of MSCs is needed when suppressing miR-383 levels in them for treating SCI.

\section{In vivo survival and limited expansion of transplanted MSCs}

In order to understand the survival and expansion of transplanted MSCs, we extracted RNA from the moues spinal tissue that had received MSCs at different time courses and examined the expression of GFP, a transgene present in the transplanted MSCs, but not in host cells. Compared with D0 (immediate sampling after transplantation), we did not find significant decrease or increase of GFP levels at D2, D7, D14 and D28, suggesting good survival and limited proliferation of MSCs in vivo for the period of this experiment (Fig. 5). To summarize, here our data suggest that upregulation of CDK19 and VEGF-A by miR-383 suppression in MSCs does not harm the therapeutic potential of MSCs in treating SCI in rats.

\section{Discussion}

Our previous report highly suggests that suppression of miR-383 may increase the therapeutic potential of human MSCs in treating SCI via augmentation of GDNF protein levels [9]. However, it is still unknown which none-GDNF genes may be affected by miR-383 suppression in MSCs, and how modulation of non-GDNF genes may affect the therapeutic effects of MSCs on SCI. This is a very important question, since alteration of unknown genes may change the overall epigenetics of MSCs, and this change may be harmful to the MSCs themselves and to the therapeutic potential of MSCs in treating SCI.

Here, after careful screening, we located 2 important genes that were altered by miR383 suppression in MSCs, and these 2 genes play critical roles in cell turn-over and cross-talk with endothelial cells, respectively.

CDK19 is previously known as CDK8-like, CDK8L or CDC2L6, due to its high amino acid sequence conservation with CDK8 [15]. CDK8 and CDK19 are found to be associate with identical mediator complexes, but appear to have non-redundant function and sometime even play opposite roles in VP16-dependent transcription, largely due to their difference in C-terminal regions [15]. As a critical factor involved in cell-cycle turnover, augmentation of CDK19 was expected to increase MSC proliferation, which was confirmed in the current study. However, this slight but significant increases in MSC proliferation should not be harmful for SCI treatment. Increases in cell growth may enhance their survival in vivo and may even reduce the number of the transplanted MSCs. Indeed, knockdown of the increases in CDK19 in MSCs-as-miR-383 did not exhibit better therapeutic effects on SCI. Hence, the upregulation of CDK19 by miR-383 suppression does not need to be further modulated in MSCs before the cells are used for transplantation to treat SCI.

VEGF-A is the most potent pro-angiogenic factor [16]. It has been acknowledged that the angiogenesis after SCI plays a role in the recovery. As early as in 2000, it was reported 
that VEGF and VEGF receptors in the spinal cord was induced after mechanical spinal injury and prostaglandin administration [17]. Later on, increases in VEGF-A have been shown to be beneficial for post-SCI recovery in most of the studies [18-21], although ineffective results have also been shown [22]. Nevertheless, adverse effects of VEGF-A have not been reported. Therefore, here we did not expect that upregulation of VEGF-A by miR-383 suppression in MSCs might be harmful to MSC-based SCI treatment. Indeed, knockdown of the increases in VEGF-A in MSCs-as-miR-383 did not exhibit better therapeutic effects on SCI. Thus, the effects of increases in the pro-angiogenic potential of MSCs-as-miR-383 that upregulates VEGF-A do not harm the therapeutic effects of MSCs-as-miR-383 on SCI, probably due to that the increases in VEGF levels in the current study are very modest and should not induce dramatic increases in vascular permeability. Hence, like CDK19, the upregulation of VEGF-A by miR-383 suppression does not need to be further modulated in MSCs before the cells are used for transplantation to treat SCI.

On the other hand, although both upregulation of CDK19 and VEGF-A in GDNFupregulated MSCs is expected to further improve the therapeutic potential of MSCs on SCI, we did not find that this change reached significance (therapeutic effect comparison between MSCs-as-miR-383 and MSCs-as-miR-383-shCDK19; therapeutic effect comparison between MSCs-as-miR-383 and MSCs-as-miR-383-shVEGF-A) besides a trend. Further increases in experimental animal number may be necessary for a detailed analysis.

\section{Acknowledgements}

This study was supported by the National Natural Science Foundation of China (Grant No. 81371368) and the Fundamental Research Funds for the Provincial Universities (Grant No. 2017LCZX29).

\section{Disclosure Statement}

No conflict of interests exists.

\section{References}

\footnotetext{
1 Li B, Shao Q Ji D, Li F, Chen G: Mesenchymal Stem Cells Mitigate Cirrhosis through BMP7 Cell Physiol Biochem 2015;35:433-440.

- Liu W, Zhang S, Gu S, Sang L, Dai C: Mesenchymal Stem Cells Recruit Macrophages to Alleviate Experimental Colitis Through TGFbeta1 Cell Physiol Biochem 2015;35:858-865.

-3 Zhang J, Wu Y, Chen A, Zhao Q: Mesenchymal stem cells promote cardiac muscle repair via enhanced neovascularization. Cell Physiol Biochem 2015;35:1219-1229.

-4 Ribeiro TB, Duarte AS, Longhini AL, Pradella F, Farias AS, Luzo AC, Oliveira AL, Olalla Saad ST: Neuroprotection and immunomodulation by xenografted human mesenchymal stem cells following spinal cord ventral root avulsion. Scientific reports 2015;5:16167.

>5 Ding Y, Zhang RY, He B, Liu Z, Zhang K, Ruan JW, Ling EA, Wu JL, Zeng YS: Combination of electroacupuncture and grafted mesenchymal stem cells overexpressing TrkC improves remyelination and function in demyelinated spinal cord of rats. Scientific reports 2015;5:9133.

6 Chen D, Zeng W, Fu Y, Gao M, Lv G: Bone marrow mesenchymal stem cells combined with minocycline improve spinal cord injury in a rat model. Int J Clin Exp Pathol 2015;8:11957-11969.

7 Lin LF, Doherty DH, Lile JD, Bektesh S, Collins F: GDNF: a glial cell line-derived neurotrophic factor for midbrain dopaminergic neurons. Science 1993;260:1130-1132.

8 Suzuki M, McHugh J, Tork C, Shelley B, Hayes A, Bellantuono I, Aebischer P, Svendsen CN: Direct muscle delivery of GDNF with human mesenchymal stem cells improves motor neuron survival and function in a rat model of familial ALS. Mol Ther 2008;16:2002-2010.
} 


\section{Cellular Physiology Cell Physiol Biochem 2018;47:129-139

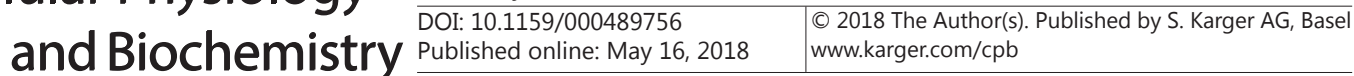 \\ Wei et al.: MiR-383-Depleted Mscs in Treating SCI}

-9 Wei GJ, An G, Shi ZW, Wang KF, Guan Y, Wang YS, Han B, Yu EM, Li PF, Dong DM, Wang LP, Teng ZW, Zhao DL: Suppression of MicroRNA-383 Enhances Therapeutic Potential of Human Bone-Marrow-Derived Mesenchymal Stem Cells in Treating Spinal Cord Injury via GDNF. Cell Physiol Biochem 2017;41:14351444.

10 Zhu P, Zhang J, Zhu J, Shi J, Zhu Q, Gao Y: MiR-429 Induces Gastric Carcinoma Cell Apoptosis Through Bcl-2 Cell Physiol Biochem 2015;37:1572-1580.

11 Zhang T, Tian F, Wang J, Jing J, Zhou SS, Chen YD: Atherosclerosis-Associated Endothelial Cell Apoptosis by MiR-429-Mediated Down Regulation of Bcl-2 Cell Physiol Biochem 2015;37:1421-1430.

12 Sun DK, Wang JM, Zhang P, Wang YQ: MicroRNA-138 Regulates Metastatic Potential of Bladder Cancer Through ZEB2 Cell Physiol Biochem 2015;37:2366-2374.

13 Song W, Li Q, Wang L, Wang L: Modulation of Fox01 Expression by miR-21 to Promote Growth of Pancreatic Ductal Adenocarcinoma. Cell Physiol Biochem 2015;35:184-190.

14 Agarwal V, Bell GW, Nam JW, Bartel DP: Predicting effective microRNA target sites in mammalian mRNAs. eLife $2015 ; 4$

15 Dale T, Clarke PA, Esdar C, Waalboer D, Adeniji-Popoola O, Ortiz-Ruiz MJ, Mallinger A, Samant RS, Czodrowski P, Musil D, Schwarz D, Schneider K, Stubbs M, Ewan K, Fraser E, TePoele R, Court W, Box G, Valenti M, de Haven Brandon A, Gowan S, Rohdich F, Raynaud F, Schneider R, Poeschke O, Blaukat A, Workman P, Schiemann K, Eccles SA, Wienke D, Blagg J: A selective chemical probe for exploring the role of CDK8 and CDK19 in human disease. Nat Chem Biol 2015;11:973-980.

16 Eichmann A, Simons M: VEGF signaling inside vascular endothelial cells and beyond. Curr Opin Cell Biol 2012;24:188-193.

17 Skold M, Cullheim S, Hammarberg H, Piehl F, Suneson A, Lake S, Sjogren A, Walum E, Risling M: Induction of VEGF and VEGF receptors in the spinal cord after mechanical spinal injury and prostaglandin administration. Eur J Neurosci 2000;12:3675-3686.

-18 Lian Jin H, Pennant WA, Hyung Lee M, Su S, Ah Kim H, Lu Liu M, Soo Oh J, Cho J, Nyun Kim K, Heum Yoon D, Ha Y: Neural stem cells modified by a hypoxia-inducible VEGF gene expression system improve cell viability under hypoxic conditions and spinal cord injury. Spine 2011;36:857-864.

19 Liu Y, Figley S, Spratt SK, Lee G, Ando D, Surosky R, Fehlings MG: An engineered transcription factor which activates VEGF-A enhances recovery after spinal cord injury. Neurobiol Dis 2010;37:384-393.

20 Patel CB, Cohen DM, Ahobila-Vajjula P, Sundberg LM, Chacko T, Narayana PA: Effect of VEGF treatment on the blood-spinal cord barrier permeability in experimental spinal cord injury: dynamic contrast-enhanced magnetic resonance imaging. J Neurotrauma 2009;26:1005-1016.

21 Kim HM, Hwang DH, Lee JE, Kim SU, Kim BG: Ex vivo VEGF delivery by neural stem cells enhances proliferation of glial progenitors, angiogenesis, and tissue sparing after spinal cord injury. PLoS One 2009;4:e4987.

22 van Neerven S, Joosten EA, Brook GA, Lambert CA, Mey J, Weis J, Marcus MA, Steinbusch HW, van Kleef M, Patijn J, Deumens R: Repetitive intrathecal VEGF(165) treatment has limited therapeutic effects after spinal cord injury in the rat. J Neurotrauma 2010;27:1781-1791. 